\title{
Left Ventricular Pseudo-aneurysm with Ventricular Septal Perforation after Acute Myocardial Infarction: a Case Report and Review of Literature
}

\author{
Muzheng $\mathrm{Li}^{1}, \mathrm{Na} \mathrm{Liu}^{1}$, Jiang Liu ${ }^{1}$, Jianjun Tang ${ }^{1}$, Xiaobo Liao ${ }^{1}$, Wanyun Zuo ${ }^{1}$, Mohan $\mathrm{Li}^{1}$, \\ and Qiming Liu ${ }^{1}$ \\ ${ }^{1}$ Second Xiangya Hospital
}

September 11, 2020

\begin{abstract}
We report a case with left ventricular pseudo-aneurysm and ventricular septal perforation after acute myocardial infarction. A 77-year-old man was admitted because of repeated chest distress and chest pain for 1 week, which was diagnosed by an electrocardiogram with acute anteroseptal ST-segment elevation myocardial infarction and troponin T elevation. The transthoracic echocardiography and cardiac magnetic resonance showed the left ventricular apical pseudo-aneurysm and lower segment of ventricular septal perforation, and confirmed by left ventriculography. Surgical treatment was performed, with operation procedure successfully. In this article, we report a rare case in clinical and review the literatures of left ventricular pseudo-aneurysm.
\end{abstract}

\section{Introduction}

Left ventricular (LV) pseudo-aneurysm is a rare disease in clinical which is mostly caused by the ventricular wall rupture after acute myocardial infarction (MI) ${ }^{[1]}$. Both LV pseudo-aneurysm and cardiac rupture are severe and fatal complications of MI, which can lead to death in a short time, and require urgent treatment. Here, we report a case with left ventricular pseudo-aneurysm and ventricular septal perforation after acute anteroseptal ST-segment elevation myocardial infarction.

\section{Case Presentation}

A 77-year-old man was admitted to the coronary care unit (CCU) of our hospital due to repeated chest distress and chest pain for 1 week, with smoking history for 50 years. He has diabetes for 10 years, which he used glimepiride and blood sugar control was acceptable currently. Physical examination showed that: normal vital signs, clear lungs, a systolic-diastolic murmur in the apex. The findings of laboratory analysis were as follows: cardiac troponin T, $382.90 \mathrm{pg} / \mathrm{ml}$ (reference range: $0^{\sim} 14 \mathrm{pg} / \mathrm{ml}$ ), creatinine kinase $(\mathrm{CK}), 36.3 \mathrm{u} / \mathrm{l}$ (reference range: $50^{\sim} 310 \mathrm{u} / \mathrm{l}$ ), CK-MB, 10.3u/l (reference range: $0^{\sim} 24 \mathrm{u} / \mathrm{l}$ ), N-terminal-pro B-type natriuretic peptide (NT-pro BNP), $4194 \mathrm{pg} / \mathrm{ml}$ (reference range: $0^{\sim} 450 \mathrm{pg} / \mathrm{ml}$ ). The electrocardiogram (ECG) revealed sinus rhythm with Q wave in leads II, III, aVF and ST-segment elevation in leads V2-V4 (Fig.1A). A chest X-ray showed cardiomegaly (cardiothoracic ratio is about 0.60) (Fig.1B). Transthoracic echocardiography revealed an aneurysm-like bulge outside the LV cavity, which measured $13^{*} 28 \mathrm{~mm}$ and was connected to the LV cavity through a small orifice (Fig.2A and B, Videos 1 and 2), with ventricular septal perforation seen in the lower segment of ventricular septum about $3 \mathrm{~mm}$ in size (Fig.2C, Video 3). The cardiac magnetic resonance (CMR) confirmed the presence of the LV pseudo-aneurysm, and showed more clearly (Fig.3A and B, Videos 4 and 5). Coronary angiography demonstrated $90 \%$ stenosis in the mid left-anterior descending artery, with totally distal occlusion. Other significant stenosis included $80 \%$ of left circumflex artery and $90 \%$ of posterior descending artery stenosis (Fig.4A, B and C, Videos 6, 7 and 8). Left ventriculography more 
intuitively showed the LV pseudo-aneurysm and ventricular septal perforation (Fig.4D, Video 9). The final diagnosis is acute anteroseptal ST-segment elevation MI with LV pseudo-aneurysm and ventricular septal perforation. In the CCU room, we gave patient oxygen and ECG monitoring and used asprin and clopidogrel to prevent platelet aggregation, atorvastatin to lower blood lipid and other anti-atherosclerotic treatment. After the patient's condition was stable, he was transferred to department of cardiovascular surgery for coronary artery bypass grafting (CABG) and excision of aneurysm (Fig.5A and B). After hospitalized for 2 months, the patient was discharged with an improvement of the disease.

\section{Discussion}

LV pseudo-aneurysm is a rare disease in clinical with a poor prognosis. It can be divided into two types: congenital and acquired ${ }^{[1]}$. The vast majority of pseudo-aneurysms are acquired, Mostly caused by infection (infective endocarditis ${ }^{[2]}$, etc.), trauma (chest penetrating injury ${ }^{[3]}$, etc.), cardiac surgery (after mitral valve or aortic valve replacement or ventricular septal defect closure ${ }^{[4-6]}$, etc.), autoimmune diseases (Behcet syndrome ${ }^{[7]}$, etc. ), but the most common reason is rupture of the ventricular wall after acute MI(maybe account for $55 \%^{[1]}$ ), which is one of the serious and fatal complications in the early stage of acute MI, often occurring within 1 week after the infarction, especially within 24 hours. At this time, due to infarcted myocardial ischemia and necrosis, the neutrophils infiltrate and soften the myocardium. With the scar tissue and fibrosis are underdeveloped, the cardiac can be perforated or ruptured under the systolic and diastolic movements and the impact of blood flow. The rupture mostly occurs in the apical, posterior and lateral walls of the left ventricle, and the incidence rate is 2 to $4 \%^{[8]}$, with usually rapid onset, leading to acute pericardial tamponade, cardiac shock and sudden death. When the rupture is diminutive, a small amount of blood flows into the pericardial cavity, stimulating the adhesion and wrapping of the myocardium and pericardium to form a bloody sac cavity, which is a pseudo-aneurysm. The width of the neck is generally less than $40 \%$ of the largest diameter of the body in pseudo-aneurysm, and there can be bidirectional blood flow between the neck and the left ventricle. Owing to the slow blood flow at the bottom of the cavity, it is easy to form thrombus and fibrous issue in LV pseudo-aneurysm.

The incidence of LV pseudo-aneurysm is low, accounting for about only $0.1 \%$ of all MI. The high risk factors include advanced age ( $>60$ years), women, hypertension, diabetes, smoking history ${ }^{[9]}$, transmural MI, lack of collateral circulation in the infarct area and delayed reperfusion therapy. The most frequently clinical manifestations are congestive heart failure, chest pain and dyspnea. A small number of patients take sudden death as the first symptom, or they can be asymptomatic $(>10 \%)^{[1]}$. cardiovascular examination can hear a systolic-diastolic murmur in the 4 and 5 intercostal space of left sternum or the base of cardiac. The ECG often shows non-specific ST segment changes or ST segment elevation. The most common X-ray's appearance is cardiomegaly.

Transthoracic echocardiography is the preferred method of examination for pseudo-aneurysm because of its non-invasive, safe, cost-effective, and bedside operation for patients with acute MI, which can accurately display the size, location, hemodynamic change and internal condition of the orifice and cavity of the LV pseudo-aneurysm as well as for pericardial effusion and pericardial tamponade, with the positive rate can reach up to $97 \%^{[10]}$. The characteristics of pseudo-aneurysm under echocardiography include: sudden interruption of myocardial echo continuity, the diameter of the orifice is significantly smaller than the maximum diameter of the cavity, and the ventricle communicates with the cavity through the orifice, there can be attached thrombus in the cavity, and the wall of pseudo-aneurysm has no myocardial structure and is easy to rupture, Color Doppler can see the multicolored blood flow bundle shuttles through the orifice to the ventricle and cavity. But when the cavity is not connected to the ventricle (such as the orifice is too small to be blocked by thrombosis or pericardial adhesion), the transthoracic echocardiography may misdiagnose pseudo-aneurysm as pericardial effusion.

CMR has a high resolution of soft tissues, which shows a clearer and more comprehensive picture of the overall situation of pseudo-aneurysm and its relationship with surrounding tissues.In addition, CMR is not affected by the limitations of the window, with multi-parameter imaging. Therefore, CMR has great significance in distinguishing true and pseudo aneurysm, as well as surgical operation and prognosis. Left ventriculography 
is the "gold standard" for the diagnosis of pseudo-aneurysm, which can clearly show the narrow and small orifice rather than the wide base of true aneurysm, but the positive rate is only $45 \%^{[10]}$. Moreover, if the injection pressure of the contrast medium is too high, it may cause the pseudo-aneurysm rupture. Coronary angiography is of great importance for the differential diagnosis and treatment of pseudo-aneurysm after MI. It can not only clearly show the culprit arteries and the whole distribution of coronary arteries, but also assess whether patients need surgical coronary artery bypass grafting (CABG) or not.

LV pseudo-aneurysm is mainly distinguished from true-aneurysm, pericardial cyst, and cardiac diverticulum. Firstly, The neck of true-aneurysm is generally large, which is equivalent to or even greater than the maximum diameter of the aneurysm. The wall of true-aneurysm has a complete ventricular wall structure, that is, endocardium, myocardial tissue, and epicardium, with coronaries distribution. True-aneurysm can occur during the acute or convalescent phase of myocardial infarction, and the rupture is infrequent and has no murmur in cardiac auscultation ${ }^{[11]}$. Secondly, Pericardial cyst mostly occurs in the right cardio phrenic angle. The transthoracic echocardiography usually shows a cystic non-echoic, smooth-walled liquid dark area outside the ventricular cavity. Color Doppler has no blood flow signal. Thirdly, cardiac diverticulum can be derived from various cardiac chambers, and it is more common in the cardiac apex. The diverticulum wall is locally bulged outward and has a systolic function, with the blood flow from the diverticulum into the ventricular cavity during the systole and opposite to the diastolic.

The mortality rate of LV pseudo-aneurysm is as high as $45 \%$ within 1 year after diagnosis ${ }^{[12]}$. For the clear diagnosis of LV pseudo-aneurysm, active treatment should be taken. According to the size and location of the neck and body of LV pseudo-aneurysm, the appropriate treatment method and timing should be cautiously selected. At present, the main treatment methods include interventional treatment and surgical operation. On the other hand, some retrospective studies have showed that cases involving chronic small LV pseudo-aneurysm of $<3 \mathrm{~cm}$ in size or patients with high surgical risk can be treated conservatively ${ }^{[13]}$.

In this article, we performed a case with simultaneous onset of LV pseudo-aneurysm and ventricular septal perforation due to acute MI, which is rarely reported before. Our coronary angiography performed three artery lesions, with $90 \%$ stenosis in the mid left-anterior descending artery and totally distal occlusion. So we can make an assumption that the LV pseudo-aneurysm and ventricular septal perforation were caused by left-anterior descending artery lesion, then CABG was performed to make the revascularization. Finally the patient was discharged and scheduled for follow-up echocardiography every 3 months after the procedure.

\section{Conclusion}

Both pseudo-aneurysm and cardiac rupture are severe and fatal mechanical complications of MI, which proper diagnosis and timely surgical operation are needed. Transthoracic echocardiography can be used as the preferred method of examination and regular follow-up echocardiography is indispensable in order to discover the recurrent pseudo-aneurysm after surgical operation.

\section{Abbreviations}

$\mathrm{LV}=$ Left ventricular

$\mathrm{MI}=$ myocardial infarction

$\mathrm{CCU}=$ coronary care unit

$\mathrm{CK}=$ creatinine kinase

NT-pro BNP $=$ N-terminal-pro B-type natriuretic peptide

$\mathrm{CMR}=$ cardiac magnetic resonance

$\mathrm{CABG}=$ coronary artery bypass grafting

$\mathrm{ECG}=$ electrocardiogram

\section{Authors' contributions}


JJT, XBL, WYZ and MHL were involved in investigation and data collection. MZL, NL, JL and QML drafted and corrected the manuscript. All authors read and approved the final manuscript for publication.

\section{Competing interests}

The authors declare that they have no conflict of interest.

\section{References}

[1] Frances C, Romero A, Grady D. Left ventricular pseudoaneurysm. J Am Coll Cardiol. 1998;32(3):557-561. doi:10.1016/s0735-1097(98)00290-3.

[2] Te Kolste HJ, Salzberg SP, Planken RN, Symersky P. Unusual complication after infective endocarditis: pseudo-aneurysm of the left ventricle. Eur Heart J. 2013;34(24):1799. doi:10.1093/eurheartj/eht143.

[3] Makaryus AN, Manetta F, Goldner B, Stephen B, Rosen SE, Park CH. Large left ventricular pseudoaneurysm presenting 25 years after penetrating chest trauma. J Interv Cardiol. 2005;18(3):193-200. doi:10.1111/j.1540-8183.2005.04057.x.

[4] Neupane S, Kommuri NV, Kazanji N, Chowdhury P. Left ventricular pseudoaneurysm after mitral valve replacement.Echocardiography. 2016;33(11):1788-1789. doi:10.1111/echo.13294.

[5] Mendiz O, Fava C, Cerda M, Lev G, Caponi G, Valdivieso L. Percutaneous repair of left ventricular pseudoaneurysm after transcatheter aortic valve replacement.Cardiovasc Revasc Med. 2017;18(6S1):3031.doi:10.1016/j.carrev.2017.02.012.

[6] Gokalp S, Ugan Atik S, Saltik IL. Percutaneous closure of a left ventricular pseudoaneurysm after transcatheter ventricular septal defect closure. Cardiol Young. 2020;30(5):743-745. doi:10.1017/S1047951120000815.

[7] Mouine N, Bennani R, Amri R. A giant left ventricular pseudoaneurysm in Behçet's disease: a case report. Cardiol Young. 2014;24(2):382-383. doi:10.1017/S1047951113000498.

[8] Zoffoli G, Mangino D, Venturini A, et al. Diagnosing left ventricular aneurysm from pseudo-aneurysm: a case report and a review in literature. J Cardiothorac Surg. 2009;4:11. Published 2009 Feb 24. doi:10.1186/1749-8090-4-11.

[9] Figueras J, Alcalde O, Barrabes JA, et al. Changes in hospital mortality rates in 425 patients with acute ST-elevation myocardial infarction and cardiac rupture over a 30-year period. Circulation. 2008;118(25):2783-2789. doi:10.1161/CIRCULATIONAHA.108.776690.

[10] Atik FA, Navia JL, Vega PR, et al. Surgical treatment of postinfarction left ventricular pseudoaneurysm. Ann Thorac Surg. 2007;83(2):526-531. doi:10.1016/j.athoracsur.2006.06.080.

[11] Antunes PE, Silva R, Ferrao de Oliveira J, Antunes MJ. Left ventricular aneurysms: early and long-term results of two types of repair. Eur J Cardiothorac Surg. 2005;27(2):210-215. doi:10.1016/j.ejcts.2004.11.010.

[12] Chandrashekar R, Konda MK, Gupta V, Kalavakunta JK. Left Ventricular Pseudoaneurysm Dissecting into the Anterior Chest Wall: A Rare Cause of Sudden Onset Excruciating Chest Pain. Eur J Case Rep Intern Med. 2017;4(1):000518. Published 2017 Jan 27. doi:10.12890/2016_000518.

[13] Alapati L, Chitwood WR, Cahill J, Mehra S, Movahed A. Left ventricular pseudoaneurysm: A case report and review of the literature. World J Clin Cases. 2014;2(4):90-93. doi:10.12998/wjcc.v2.i4.90.

\section{Figure legends}

Figure $1 \mathrm{~A}$ and B: Electrocardiogram and chest X-ray. The electrocardiogram showed normal sinus rhythm with Q wave in leads II, III, aVF and ST-segment elevation in leads V2-V4. The chest X-ray showed cardiomegaly (cardiothoracic ratio is about 0.60 ) and mild pulmonary congestion. 
Figure $2 \mathrm{~A}, \mathrm{~B}$ and C: Transthoracic echocardiography on hospital admission. The echocardiography revealed an apical LV pseudo-aneurysm (white arrows) and ventricular septal perforation (black arrow) in the apical 4-chamber view and parasternal short axis view. Videos 1, 2 and 3 correspond to figure 2. Abbreviations: LA, left atrium; LV, left ventricular; RA, right atrium; RV, right ventricular

Figure $3 \mathrm{~A}$ and $\mathrm{B}$ : Cardiac magnetic resonance imaging. The CMR confirmed the presence of the LV pseudo-aneurysm (white arrows). Videos 4 and 5 correspond to figure 3.

Figure 4 A, B, C and D: Coronary angiography and Left ventriculography. $90 \%$ stenosis was observed in the mid left-anterior descending artery, other significant stenosis was $80 \%$ of left circumflex artery and $90 \%$ of posterior descending artery stenosis (black arrows). It is clearly to be seen the LV apical pseudo-aneurysm (black arrow) and the contrast medium flows from the left ventricle into the right ventricle through the ventricular septal perforation (white arrow). Videos 6, 7, 8 and 9 correspond to figure 4. Abbreviations: CRA, cranial; LAD, left anterior descending; LAO, left anterior oblique; LCX, left circumflex; LM, left main; RCA, right coronary artery

Figure 5 A and B: Surgical procedure. We found the LV pseudo-aneurysm in the apex, and finally surgical excision and suture was performed successfully (black arrows).

\section{Supplementary Material}

Additional supplementary material may be found online.

Videos 1, 2 and 3. Transthoracic echocardiography on hospital admission. The echocardiography revealed an apical LV pseudo-aneurysm and ventricular septal perforation in the apical 4-chamber view and parasternal short axis view.

Videos 4 and 5. Cardiac magnetic resonance imaging. The CMR confirmed the presence of the LV pseudoaneurysm.

Videos 6, 7, 8 and 9. 90\% stenosis was observed in the mid left-anterior descending artery, other significant stenosis was $80 \%$ of left circumflex artery and $90 \%$ of posterior descending artery stenosis. It is clearly to be seen the LV apical pseudo-aneurysm and the contrast medium flows from the left ventricle into the right ventricle through the ventricular septal perforation.

\section{Hosted file}

Figure1.A(1).eps available at https://authorea.com/users/356804/articles/479606-leftventricular-pseudo-aneurysm-with-ventricular-septal-perforation-after-acute-myocardialinfarction-a-case-report-and-review-of-literature

\section{Hosted file}

Figure1.B(1).eps available at https://authorea.com/users/356804/articles/479606-leftventricular-pseudo-aneurysm-with-ventricular-septal-perforation-after-acute-myocardialinfarction-a-case-report-and-review-of-literature 


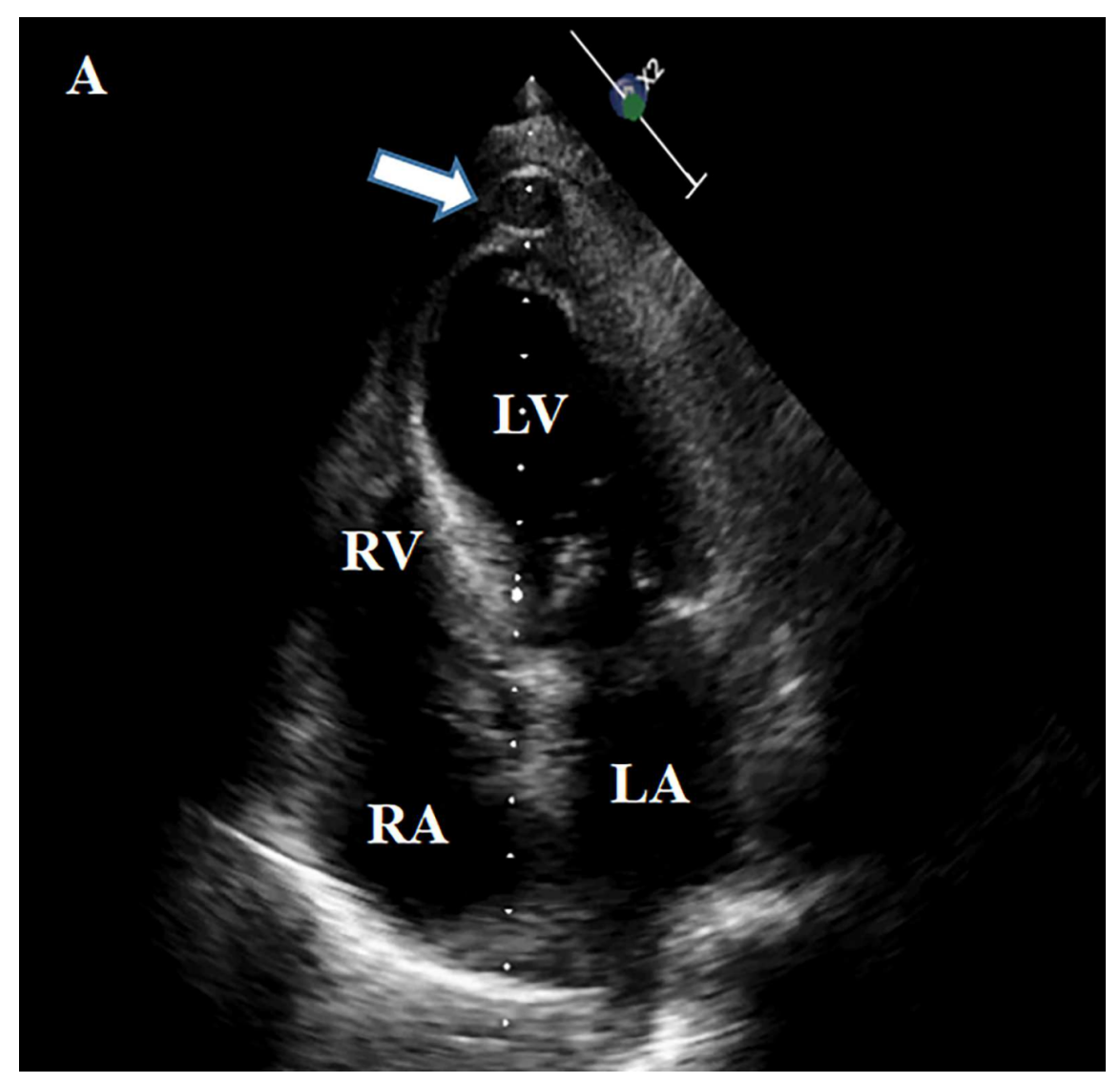



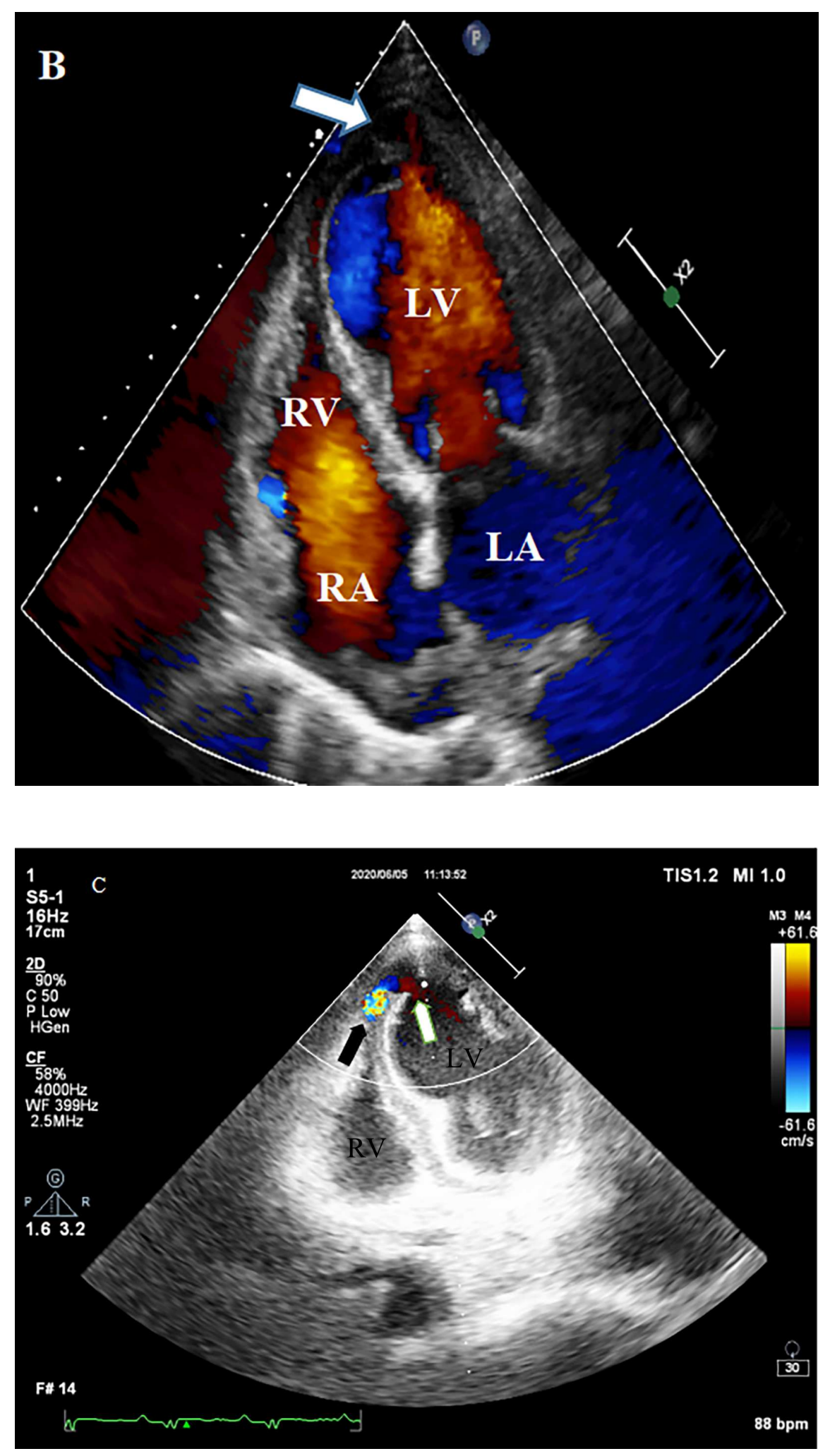

Hosted file 
Figure3.A(1).eps available at https://authorea.com/users/356804/articles/479606-leftventricular-pseudo-aneurysm-with-ventricular-septal-perforation-after-acute-myocardialinfarction-a-case-report-and-review-of-literature

\section{Hosted file}

Figure3.B(1).eps available at https://authorea.com/users/356804/articles/479606-leftventricular-pseudo-aneurysm-with-ventricular-septal-perforation-after-acute-myocardialinfarction-a-case-report-and-review-of-literature

\section{Hosted file}

Figure4.A(1).eps available at https://authorea.com/users/356804/articles/479606-leftventricular-pseudo-aneurysm-with-ventricular-septal-perforation-after-acute-myocardialinfarction-a-case-report-and-review-of-literature

\section{Hosted file}

Figure4.B(1).eps available at https://authorea.com/users/356804/articles/479606-leftventricular-pseudo-aneurysm-with-ventricular-septal-perforation-after-acute-myocardialinfarction-a-case-report-and-review-of-literature

\section{Hosted file}

Figure4.C(1).eps available at https://authorea.com/users/356804/articles/479606-leftventricular-pseudo-aneurysm-with-ventricular-septal-perforation-after-acute-myocardialinfarction-a-case-report-and-review-of-literature

\section{Hosted file}

Figure4.D(1).eps available at https://authorea.com/users/356804/articles/479606-leftventricular-pseudo-aneurysm-with-ventricular-septal-perforation-after-acute-myocardialinfarction-a-case-report-and-review-of-literature

\section{Hosted file}

Figure5.A(1).eps available at https://authorea.com/users/356804/articles/479606-leftventricular-pseudo-aneurysm-with-ventricular-septal-perforation-after-acute-myocardialinfarction-a-case-report-and-review-of-literature

\section{Hosted file}

Figure5.B(1).eps available at https://authorea.com/users/356804/articles/479606-leftventricular-pseudo-aneurysm-with-ventricular-septal-perforation-after-acute-myocardialinfarction-a-case-report-and-review-of-literature 